\title{
Effectiveness of Netarsudil as an Additional Therapy for Glaucoma in Patients Already on Maximally Tolerated Medical Therapy
}

\section{Natacha C Villegas \\ Wen-Shin Lee}

Department of Ophthalmology, Stanford University School of Medicine, Stanford, CA, USA
Correspondence: Natacha C Villegas Department of Ophthalmology, Stanford University, Medical Center, 2452 Watson Court, Palo Alto, CA, 94303-5353, USA Tel +I 832 477-3498

Email natachav@stanford.edu
Purpose: This study seeks to evaluate the effectiveness of netarsudil (Rhopressa) in patients with inadequately controlled IOP on otherwise maximally tolerated medical therapy.

Methods: This is a retrospective study of patients started on netarsudil at Stanford University. Exclusion criteria included glaucoma surgery or laser within 6 months of starting netarsudil and other modifications to the baseline medication regimen within 4 weeks of starting netarsudil. The primary outcome was treatment success, defined as IOP reduction meeting a predetermined target, and no further medication, laser, or surgery recommended subsequent to starting netarsudil.

Results: Sixty-two eyes were included, and 36 (58\%) achieved treatment success at first follow-up. Mean baseline IOP was $19.5 \pm 5.6 \mathrm{mmHg}$ on a mean of $3.5 \pm 0.7$ ocular hypotensive medications. The mean change in IOP from baseline to first follow-up was $-3.53 \mathrm{mmHg}(-17 \%)$. In patients who achieved treatment success, mean IOP change was $-5.22 \mathrm{mmHg}(-28.0 \%)$. Of the eyes with baseline IOP $\leq 20 \mathrm{mmHg}, 69 \%$ achieved treatment success, compared to only $17 \%$ of eyes with baseline IOP $\geq 21 \mathrm{mmHg}(\mathrm{P}<0.05)$.

Conclusion: Netarsudil is effective in lowering IOP for patients on otherwise maximally tolerated medical therapy, for which glaucoma laser or surgery would have been the only remaining therapeutic options. Treatment success was more likely in eyes with baseline IOP under $20 \mathrm{mmHg}$.

Keywords: netarsudil, Rhopressa, maximally tolerated medical therapy, glaucoma

\section{Introduction}

Glaucoma is the second-leading cause of blindness worldwide, with estimated 111.8 million individuals affected by $2040 .{ }^{1}$ Although the pathophysiology of this disease is thought to be multifactorial, intraocular pressure (IOP) is currently the only modifiable risk factor to slow glaucoma progression. ${ }^{2}$ In 1996, the prostaglandin analogue latanoprost was approved by the US Food and Drug Administration and quickly became a mainstay in glaucoma treatment alongside topical beta blockers, alpha agonists, and carbonic anhydrase inhibitors. It would be over 20 years until other medical therapies for glaucoma were approved for use in the US market: latanoprostene bunod (Vyzulta, Bausch and Lomb, Rochester, NY) and netarsudil (Rhopressa, Aerie Pharmaceuticals, Bedminster, NJ). ${ }^{3}$ Although prior to this, the existing topical medications for glaucoma were overall effective and well tolerated, these new medications were seen as welcome additions for patients who could not tolerate the existing topical therapies due to adverse effects, or patients for whom the existing medical therapies did not result in sufficient IOP lowering. 
Netarsudil inhibits a Rho-associated protein kinase (ROCK), leading to expansion of the juxtacanalicular trabecular meshwork and dilation of the episcleral veins, which in turn results in increased outflow facility through the conventional outflow pathway. ${ }^{4}$ Currently, netarsudil is the only medical therapy for glaucoma that directly targets outflow resistance through the conventional outflow pathway-pilocarpine has an indirect effect on the trabecular meshwork via pupillary constriction.

The ROCKET- 1 and ROCKET-2 trials demonstrated that netarsudil is a well-tolerated topical medication that significantly lowers IOP with non-inferiority when compared to timolol. ${ }^{5}$ However, this non-inferiority was demonstrated for a very specific patient demographic: those with either primary open-angle glaucoma (POAG) or ocular hypertension (OHT) with a washout IOP of $<25 \mathrm{mmHg}$, a maximum of 2 topical medications at baseline, and no history of glaucoma surgery or laser. ${ }^{7}$ Subsequently, the ROCKET-4 trial demonstrated the non-inferiority of netarsudil compared to timolol for patients with a baseline washout IOP of $<30 \mathrm{mmHg}{ }^{6}$ This was in contrast to a prior study demonstrating that netarsudil was non-inferior for patients with a baseline washout IOP of $<26 \mathrm{mmHg}$, but not as effective in lowering IOP compared to latanoprost for patients with a washout IOP $<35 \mathrm{mmHg}^{7}$

Thus, the pivotal trials for netarsudil demonstrated that it was an effective primary therapy for POAG and OHT, with comparable effectiveness to timolol and latanoprost for a specific patient population. However, few studies exist on the effectiveness of netarsudil on patients with secondary open-angle glaucoma or angle closure glaucoma, patients already on $>2$ IOP lowering medications, or patients with a history of glaucoma laser or surgery. ${ }^{8-14}$ These questions are particularly relevant, as netarsudil is less often used as an initial primary therapy owing to a high rate of conjunctival hyperemia, challenges with insurance coverage, and the existence of multiple drug classes with at least equivalent effectiveness and fewer adverse effects. It is more commonly used in clinical practice as a supplemental therapy for patients already on multiple topical medications and patients with inadequately controlled IOP despite prior glaucoma laser or surgery; clinical scenarios for which the pivotal trials do not offer clear data. This study seeks to better inform clinicians on the effectiveness of netarsudil in the clinical scenarios for which it is commonly used in real-world practice.

\section{Methods}

We conducted a retrospective chart review of patients started on netarsudil by glaucoma faculty at the Byers Eye Institute at Stanford University. This study was approved by Stanford University's Institutional Review Board. Protocols were compliant with the Health Insurance Portability and Accountability Act of 1996.

The Stanford Research Repository (STARR), an institution-wide electronic data warehouse repository of all electronic medical records at Stanford since 1998, was used to identify patients who were determined by the treating physician to require additional IOP-lowering treatment with netarsudil $0.02 \%$ once daily. Requirements for written consent were waived because of the retrospective nature of the study. For each study subject, right and left eyes were independently assessed for eligibility and IOP outcomes. Electronic medical records were reviewed to confirm eligibility of subjects, and all variables were manually extracted.

Baseline data was obtained including age, race, sex, subtype of glaucoma, severity of glaucoma based on the 10th Revision of the International Statistical Classification of Diseases diagnostic criteria, number of IOP-lowering medications used at the time netarsudil was added, and history of glaucoma surgery or laser. All sub-types of glaucoma and all pre-treatment IOPs were included. Exclusion criteria included a history of glaucoma surgery or laser within 6 months of starting netarsudil, starting or stopping another glaucoma medication within 4 weeks of starting netarsudil and until the first post-treatment follow-up visit. Patients who discontinued netarsudil before the first follow-up visit due to adverse effects were not included in this study.

The primary outcome measure of this study was treatment success, defined as (a) IOP reduction from baseline to a patient-specific IOP target predetermined by the treating physician, and (b) no further medication, laser, or surgery recommended subsequent to starting netarsudil. IOP was measured by Goldmann applanation tonometry by a physician, per standard practice at this institution. Secondary outcome measures included mean IOP reduction, relative IOP reduction from baseline, and adverse effects secondary to netarsudil. Medicationrelated adverse effects were captured and analyzed only if documented in the medical record as symptomatic by the patient. Adherence was assessed in clinical practice and was determined by patient confirmation of medication use in response to questioning by the treating physician. 
Study outcomes were summarized using means, standard deviations, and proportions, as appropriate. The change in IOP was calculated as the difference between IOP at the visit when netarsudil was first prescribed and the IOP at the first follow-up visit after this. Statistical analysis was performed using the chi-square test and 2 -sample $t$-test with a significance level of 0.05 . Power calculations of the comparison of 2 means was calculated with a significance level of 0.05 and an effect size of 0.5 .

\section{Results}

\section{Baseline Demographic and Clinical Characteristics}

Netarsudil, an adjunctive ocular hypotensive therapy, was prescribed to 45 patients (62 eyes) at the Byers Eye Institute, who met the inclusion criteria, as identified via STARR. Patients had no glaucoma surgery or laser within 6 months of starting netarsudil, and no change otherwise in their baseline glaucoma medication regimen within 4 weeks of starting netarsudil and until the first follow-up visit. Mean age in years was $61 \pm 15$, and $45 \%$ were female (Table 1). Most patients had a diagnosis of POAG (59\%), with the remainder of patients representing a broad spectrum of glaucoma subtypes, and most eyes were on $\geq 3$ ocular hypotensive medications at baseline (Table 1). The patient population included a diverse set of ethnic backgrounds (Table 1).

\section{Treatment Success}

Treatment success was achieved in 36 (58\%) eyes at the first follow-up visit after starting netarsudil, which occurred at a mean of $4.8 \pm 3.5$ weeks. Of those who achieved treatment success at the first follow-up, 27 (75\%) were still using netarsudil with treatment success at the end of the study period, with a mean follow-up time of $33 \pm 22$ weeks.

Of the eyes that achieved treatment success at first followup but were no longer on netarsudil at the end of the study period, reasons for discontinuation of netarsudil included intolerance due to adverse effects (4 eyes), having glaucoma surgery despite IOP decrease that was initially deemed sufficient (1 eye), insurance coverage issues ( 1 eye), and subsequent IOP increase to an unacceptable level ( 2 eyes).

In eyes with a baseline IOP of $\leq 20 \mathrm{mmHg}, 69 \%$ achieved treatment success, with a mean IOP change of $-2.80 \mathrm{mmHg}(-17 \%)$. Conversely, only $17 \%$ of patients
Table I Demographics and Baseline Characteristics

\begin{tabular}{|c|c|}
\hline Characteristics & Number of Eyes, n (\%) \\
\hline Age (years $\pm S D$ ) & $61 \pm 15$ \\
\hline \multicolumn{2}{|l|}{ Sex } \\
\hline Female & $28(45)$ \\
\hline Male & $34(54)$ \\
\hline \multicolumn{2}{|l|}{ Types of glaucoma } \\
\hline POAG & $37(59)$ \\
\hline PXG & $4(6)$ \\
\hline MMG & $5(8)$ \\
\hline NTG & $3(4)$ \\
\hline PDS & $3(4)$ \\
\hline CACG & $2(3)$ \\
\hline JOAG & $2(3)$ \\
\hline PACG & $2(3)$ \\
\hline Aphakic & $2(3)$ \\
\hline Angle recession & $\mathrm{I}(\mathrm{I})$ \\
\hline \multicolumn{2}{|l|}{ Race } \\
\hline East Asian & $19(30)$ \\
\hline White & $20(32)$ \\
\hline Hispanic & II (17) \\
\hline Black & $6(9)$ \\
\hline South Asian & $4(6)$ \\
\hline Unknown & $2(3)$ \\
\hline \multicolumn{2}{|l|}{ Number of baseline medications } \\
\hline$\leq 2$ & $5(8)$ \\
\hline 3 & $19(30)$ \\
\hline$\geq 4$ & $38(6 \mathrm{I})$ \\
\hline \multicolumn{2}{|l|}{ Glaucoma severity } \\
\hline Mild & $8(12)$ \\
\hline Moderate & $10(16)$ \\
\hline Severe & $42(67)$ \\
\hline \multicolumn{2}{|l|}{ Medication Classes } \\
\hline Prostaglandin analog & $57(9 I)$ \\
\hline Beta-adrenergic antagonist & $53(85)$ \\
\hline Alpha-2 agonist & $48(77)$ \\
\hline Carbonic anhydrase inhibitor & $54(87)$ \\
\hline Cholinergic agonist & I (I) \\
\hline \multicolumn{2}{|l|}{ Surgical glaucoma procedures } \\
\hline Glaucoma drainage implant & $4(6)$ \\
\hline Trabeculectomy & $\mathrm{I}(\mathrm{I})$ \\
\hline Trabeculotomy & $11(17)$ \\
\hline MIGS & $5(8)$ \\
\hline Trans-scleral CPC & 0 \\
\hline LPI & $3(4)$ \\
\hline Laser trabeculoplasty & $8(12)$ \\
\hline
\end{tabular}

Abbreviations: SD, standard deviation; POAG, primary open angle glaucoma; PXG, pseudoexfoliation glaucoma; MMG, mixed mechanism glaucoma; NTG, normal tension glaucoma; CACG, pigment dispersion glaucoma, chronic angle closure glaucoma; JOAG, juvenile open angle glaucoma; PACG, primary angle closure glaucoma; MIGS, minimally invasive glaucoma surgery; CPC, cyclophotocoagulation; LPI, laser peripheral iridotomy. 
with baseline IOP $\geq 21 \mathrm{mmHg}$ achieved treatment success, with a mean IOP change of $-5.05 \mathrm{mmHg}(-18.73 \%)$. This difference was statistically significant $(\mathrm{P}=0.01)$.

In the 14 eyes with a baseline history of trabeculectomy or glaucoma tube shunt implant prior to starting netarsudil, 6 eyes (42\%) achieved treatment success. The mean change in the IOP for this cohort was $-2.70 \mathrm{mmHg}$ $(-13.9 \%)$. The difference in treatment success between eyes with and without a history of glaucoma incisional surgery was not statistically significant. However, the power of this calculation was not reached.

Inter-eye correlation was accounted for in a unit analysis per subject rather than eyes, choosing the right eye in patients with both eyes enrolled in the study. In this analysis, we found that 28 of 45 patients $(62.2 \%)$ achieved treatment success, similar to the $58 \%$ found when considering all 62 eyes in the cohort.

\section{Change in IOP}

The mean IOP at the time of starting netarsudil was $19.4 \pm 5.6$ $\mathrm{mmHg}$ on an average of 3.5 medications. The mean change in IOP for all patients from baseline to the first follow-up was $-3.53 \mathrm{mmHg}(-17 \%)$, resulting in a mean post-treatment IOP of $16.0 \pm 5.8 \mathrm{mmHg}(\mathrm{p}<0.05)$. In patients who achieved treatment success, the mean change in IOP was $-5.22 \mathrm{mmHg}$ $(-28.0 \%)(\mathrm{p}<0.05)$. In patients who failed treatment, the mean change in IOP was $-1.19 \mathrm{mmHg}(-3.42 \%)$.

The majority of eyes were on maximally tolerated medical therapy (MTMT), with 54 eyes (87\%) being treated with $\geq 3$ topical medications. Most of the patients not on 4 topical medications had either intolerable adverse effects or no treatment response to the medications not being utilized. Lowering of IOP was statistically significant regarless of the number of baseline medications (Table 2). Patients with 3 IOP-lowering medications at baseline had a decrease of $4.26 \mathrm{mmHg}$ versus 2.83 $\mathrm{mmHg}$ in the group with 4 medications, likely driven by two outliers in the cohort with 3 baseline medications, one with IOP decrease of $12 \mathrm{mmHg}$ and another of $16 \mathrm{mmHg}$.

Table 2 Change in IOP Stratified by Number of Baseline Medications

\begin{tabular}{|l|c|c|}
\hline $\begin{array}{l}\text { No. of Baseline } \\
\text { Medications }\end{array}$ & $\begin{array}{c}\text { Patients } \\
\text { (n) }\end{array}$ & $\begin{array}{c}\text { IOP Decrease in mmHg } \\
\text { (P-value) }\end{array}$ \\
\hline$\leq 2$ & $5(8)$ & $4(0.001)$ \\
3 & $19(30)$ & $4.26(<0.001)$ \\
$\geq 4$ & $38(6 I)$ & $2.83(<0.001)$ \\
\hline
\end{tabular}

P-value was still significant after analysis without these two patients and showed an IOP decrease of $3.11 \mathrm{mmHg}$. Reduction in IOP did not depend significantly on glaucoma severity or whether patients underwent prior glaucoma surgical interventions.

In 17 eyes (27\%), IOP actually increased or did not change from the pre-treatment baseline. In patients in whom IOP decreased with netarsudil, the percentage change in IOP followed a normal distribution centered around the $21-30 \%$ range with $48.3 \%$ of patients achieving an IOP reduction of $>20 \%, 25.8 \%$ over $30 \%$, and $12.9 \%$ over $40 \%$.

Of the patients who were still on netarsudil at last follow-up ( $33 \pm 22$ weeks), IOP was maintained at goal throughout the follow-up period of the study for all but 2 patients, suggesting a low incidence of tachyphylaxis.

\section{Glaucoma Surgery During Study Follow-Up Period}

All 26 eyes that failed treatment with netarsudil were offered glaucoma surgery, with $13(50 \%)$ of these eyes undergoing glaucoma surgery by the end of the study period. An additional $6(16 \%)$ eyes with initial treatment success with netarsudil had glaucoma surgery by the end of the study period. The difference in the rate of glaucoma surgery between eyes that achieved initial treatment success and those who did not was statistically significant $(\mathrm{p}=0.004)$.

The primary reason for not proceeding with glaucoma surgery in patients who failed treatment was the patient's refusal to undergo surgery despite recommendations to do so (10 eyes). In other cases, visual fields were stable despite IOP not at target ( 2 eyes), and in one patient surgery had been planned but not yet performed at the end of the study period.

For the 6 patients who had glaucoma surgery despite initial treatment success, surgery was performed due to treatment failure at subsequent visits ( 3 eyes), discontinuation of netarsudil due to adverse effects ( 1 eye), subsequent minimally invasive glaucoma surgery (MIGS) at the time of routine cataract extraction ( 1 eye), and in one eye surgery was performed despite treatment success per patient's request.

\section{Adverse Effects}

A total of $20 \%$ of patients self-reported an adverse effect after starting netarsudil. The most common adverse effects were conjunctival hyperemia ( $12.9 \%$ of eyes) and ocular surface irritation $(6.4 \%$ of eyes) (Table 3$)$. Other less common adverse effects included pruritus, cornea verticillata, glare and halos. Adverse effects were significant 
Table 3 Adverse Effects

\begin{tabular}{|l|c|}
\hline Adverse Effects & N (\%) \\
\hline Total & $13(20.9)$ \\
Discontinued after first follow-up & $4(6.4)$ \\
Injection & $8(12.9)$ \\
Irritation & $7(11.2)$ \\
Pruritus & $2(3.2)$ \\
Not specified & $2(3.2)$ \\
Verticillata & $1(1.6)$ \\
Glare/halo & $1(1.6)$ \\
\hline
\end{tabular}

enough to lead to discontinuation of netarsudil in $4(6 \%)$ of the patients who had initially met the treatment goal.

\section{Discussion}

In this study, netarsudil was found to be effective in significantly lowering IOP for patients already on MTMT. Moreover, it was successful in achieving a pre-specified target IOP in $58 \%$ of eyes for which glaucoma laser or surgery would be the only remaining therapeutic option in the absence of this drug. In these eyes, the IOP reduction was on average a robust $5.22 \mathrm{mmHg}$, or $28 \%$ from pretreatment baseline, which is clinically significant as it resulted in a significantly reduced likelihood of undergoing glaucoma surgery during the study period.

Similar to our findings, pivotal clinical trials ROCKET-1 and ROCKET- 2 showed a mean reduction in IOP with netarsudil $0.02 \%$ daily that ranged between $3.3 \mathrm{mmHg}$ and 4.6 $\mathrm{mmHg}$, in patients with baseline IOP $<25 \mathrm{mmHg}{ }^{5}$ A retrospective study by Prager et al of 175 eyes found similar magnitudes of IOP reduction after adding netarsudil as an adjunctive therapy, regardless of the baseline IOP or the number of medications in use at baseline, with a $3.0 \mathrm{mmHg}$ IOP decrease in patients with 3 medications, and a $2.9 \mathrm{mmHg}$ decrease in patients with 4 medications. ${ }^{8}$ Furthermore, Mehta et al conducted a retrospective study with 95 patients that found a mean reduction in IOP of $3.9 \pm 4.6 \mathrm{mmHg}$ after starting netarsudil, which also did not depend on baseline number of IOP-lowering medications. ${ }^{9}$ Other studies have also demonstrated significant decreases in IOP regardless of the number of IOP-lowering medications. ${ }^{10-14}$

In our study, $6 \%$ of eyes discontinued netarsudil due to adverse effects after the first follow-up visit (Table 3), compared to $10 \%$ and $12 \%$ in ROCKET- 1 and ROCKET-2 trials, respectively. ${ }^{7}$ This lower incidence in our study is likely due to only capturing adverse effects reported by symptomatic patients, given the lack of an adverse effect questionnaire in this retrospective study. Moreover, most of our patients were already on multiple topical medications at baseline, including prostaglandin analogues, which may have resulted in a baseline degree of conjunctival hyperemia. Of note, we only included patients who were still on netarsudil on the first followup visit. Patients who discontinued netarsudil due to adverse effects before the first follow-up visit were not included in the analysis. This may also explain the lower incidence of adverse effects in our study.

The onset of hyperemia is thought to be the result of a reduction in myosin light-chain phosphorylation of vascular smooth muscle cells and disinhibition of endothelial nitric oxide synthase. ${ }^{15}$ As prior literature has demonstrated reduced incidence and severity of conjunctival hyperemia with repeated dosing, it may be prudent to continue netarsudil for patients with this finding if they have achieved good IOP reduction and are tolerating netarsudil otherwise. ${ }^{16}$

Our results indicate that netarsudil is more likely to successfully achieve a pre-specified target IOP when baseline IOP is $\leq 20 \mathrm{mmHg}$. The reason for this may be multifactorial. First, it is likely that patients with a lower baseline IOP require a smaller absolute reduction in IOP in order to meet their IOP target. Secondly, it is possible that eyes with a very high IOP despite otherwise MTMT have more severe aqueous outflow pathway dysfunction that is less likely to respond to netarsudil. Finally, netarsudil's possible effect on episcleral venous pressure may lead to increased effectiveness in eyes with lower baseline IOP. These findings suggest that clinicians should temper their expectations when adding netarsudil in patients with a baseline IOP of $\geq 21 \mathrm{mmHg}$.

The limitations of this study are primarily those inherent to a retrospective study, including non-uniform follow-up, no control cohort, IOP measurement times and drop instillation times were haphazard, and the possibility of inconsistent medication compliance. Indications for initiation of netarsudil were at the treating physician's discretion. Furthermore, treatment success was defined as whether IOP decreased below a predetermined, patient-specific target, or if no further medication, laser, or surgery was recommended. This threshold was decided in a non-standardized, patientspecific manner rather than a uniform study protocol. However, patients who achieved treatment success in our study achieved a robust reduction in IOP, which may serve as a more broadly applicable measure of success. Finally, regression to the mean is a possibility in this study given that the analysis included only one baseline IOP measurement. 


\section{Conclusion}

Adding netarsudil $0.02 \%$ once daily to glaucoma patients with inadequate IOP reduction on otherwise maximally tolerated medical therapy can result in significant IOP reduction, thus deferring the need for glaucoma laser or incisional surgery. Treatment success under these circumstances may be more likely when baseline IOP is $\leq 20 \mathrm{mmHg}$.

\section{Acknowledgment}

The study was presented in the form of a poster at the 2021 Association for Research in Vision and Ophthalmology annual meeting.

\section{Disclosure}

The authors report no conflicts of interest in this work.

\section{References}

1. Tham YC, Li X, Wong TY, Quigley HA, Aung T, Cheng CY. Global prevalence of glaucoma and projections of glaucoma burden through 2040: a systematic review and meta-analysis. Ophthalmology. 2014;121(11):2081-2090. doi:10.1016/j.ophtha.2014.05.013

2. Leske MC, Heijl A, Hyman L, Bengtsson B. Early manifest glaucoma trial: design and baseline data. Ophthalmology. 1999;106 (11):2144-2153. doi:10.1016/S0161-6420(99)90497-9

3. Mehran NA, Sinha S, Razeghinejad R. New glaucoma medications: latanoprostene bunod, netarsudil, and fixed combination netarsudil-latanoprost. Eye. 2020;34(1):72-88. PMID: 31695162; PMCID: PMC7002400. doi:10.1038/s41433-019-0671-0

4. Ren R, Li G, Le TD, Kopczynski C, Stamer WD, Gong H. Netarsudil increases outflow facility in human eyes through multiple mechanisms. Invest Ophthalmol Vis Sci. 2016;57(14):6197-6209. PMID: 27842161; PMCID: PMC5114035. doi:10.1167/iovs.16-20189

5. Serle JB, Katz LJ, McLaurin E, et al; ROCKET-1 and ROCKET-2 Study Groups. Two Phase 3 clinical trials comparing the safety and efficacy of netarsudil to timolol in patients with elevated intraocular pressure: rho kinase elevated iop treatment trial 1 and 2 (ROCKET-1 and ROCKET-2). Am J Ophthalmol. 2018;186:116-127. Epub 2017 Dec 1. PMID: 29199013. doi:10.1016/j.ajo.2017.11.019
6. Khouri AS, Serle JB, Bacharach J, et al; Rocket-4 Study Group. Once-daily netarsudil versus twice-daily timolol in patients with elevated intraocular pressure: the randomized phase 3 ROCKET-4 Study. Am J Ophthalmol. 2019;204:97-104. PMID: 30862500. doi:10.1016/j.ajo.2019.03.002

7. Bacharach J, Dubiner HB, Levy B, Kopczynski CC, Novack GD; AR-13324-CS202 Study Group. Double-masked, randomized, dose-response study of AR-13324 versus latanoprost in patients with elevated intraocular pressure. Ophthalmology. 2015;122 (2):302-307. doi:10.1016/j.ophtha.2014.08.022

8. Prager AJ, Tang M, Pleet AL, Petito LC, Tanna AP. Effectiveness and tolerability of netarsudil in combination with other ocular hypotensive agents. Ophthalmol Glaucoma. 2021. doi:10.1016/j. ogla.2021.03.014

9. Mehta AA, Kanu LN, Sood-Mendiratta S, et al. Experience with netarsudil $0.02 \%$ and latanoprostene bunod $0.024 \%$ as adjunctive therapy for glaucoma. Eur J Ophthalmol. 2021;1120672121998913. doi:10.1177/1120672121998913

10. Ustaoglu M, Shiuey E, Sanvicente C, et al. The efficacy and safety profile of netarsudil $0.02 \%$ in glaucoma treatment: real-world outcomes. Invest Ophthalmol Vis Sci. 2019;60:2393.

11. Tang M, Prager A, Pleet A, et al. Retrospective analysis of the efficacy and safety of netarsudil in clinical practice. Invest Ophthalmol Vis Sci. 2020;61:1241.

12. Woolf S, Bahr T, Waldman C. The addition of latanoprostene bunod or netarsudil to glaucoma patients on standard maximum medical therapy. Invest Ophthalmol Vis Sci. 2020;61:1224.

13. Mehta P, Kaplowitz K, Lenoci J, et al. IOP lowering efficacy of adjunctive netarsudil (Rhopressa): a retrospective chart review. Invest Ophthalmol Vis Sci. 2020;61:1237.

14. Lin MM, Moster SJ, Zheng CX, et al. Netarsudil's effect in eyes with a history of selective laser trabeculoplasty. Ophthalmol Glaucoma. 2020;3(4):306-308. doi:10.1016/j.ogla.2020.01.005

15. Ming X-F, Viswambharan H, Barandier C, et al. Rho GTPase/Rho kinase negatively regulates endothelial nitric oxide synthase phosphorylation through the inhibition of protein kinase B/Akt in human endothelial cells. Mol Cell Biol. 2002;22:8467-8477. doi:10.1128/ MCB.22.24.8467-8477.2002

16. Weiss M, Levy B, Kopczynski C, et al. Evaluation of AR-13324, a novel dual mechanism agent, in lowering of IOP in glaucoma and ocular hypertension. Investig Ophthalmol Vis Sci. 2013;54(15):754.
Clinical Ophthalmology

\section{Publish your work in this journal}

Clinical Ophthalmology is an international, peer-reviewed journal covering all subspecialties within ophthalmology. Key topics include: Optometry; Visual science; Pharmacology and drug therapy in eye diseases; Basic Sciences; Primary and Secondary eye care; Patient Safety and Quality of Care Improvements. This journal is indexed on PubMed
Central and CAS, and is the official journal of The Society of Clinical Ophthalmology (SCO). The manuscript management system is completely online and includes a very quick and fair peer-review system, which is all easy to use. Visit http://www.dovepress.com/ testimonials.php to read real quotes from published authors.

\section{Dovepress}

